\title{
Percepções de um grupo de voluntários frente ao trabalho com pacientes oncológicos
}

\author{
Perceptions of a group of volunteers of the work \\ with oncological patients
}

Adriana Cândida de Souza ${ }^{1}$, Andréa Cristina Alves², Gabriela da Cunha Januário ${ }^{3}$, Marilene Elvira de Faria Oliveira ${ }^{4}$, Maria Inês Lemos Coelho Ribeiro ${ }^{5}$, Aline Teixeira Silva ${ }^{6}$

\begin{abstract}
1. Enfermeira. Especialista em Enfermagem Oncológica. Enfermeira do Hospital do Câncer de Passos - MG. E-mail: adrianacandidadesouza@gmail.com ORCID: http://orcid.org/0000-0002-3483-1086

2.Enfermeira. Mestre em Enfermagem. Docente no Instituto Federal do Sul de Minas - IFSULDEMINAS. E-mail: andrea.alves@ifsuldeminas.edu.br ORCID: http://orcid.org/0000-0003-1535-4832

3. Enfermeira. Mestre em Enfermagem. Docente na Universidade do Estado de Minas Gerais - UEMG. E-mail: gabriela_cunha92@hotmail.com ORCID: http://orcid.org/0000-0002-3585-0705

4.Enfermeira. Mestre em Enfermagem. Atuante no SAMU do Sul de Minas. E-mail: marilenefaria30@yahoo. com.br ORCID: http://orcid.org/0000-0003-0962-0129

5.Enfermeira. Doutora em Enfermagem. Docente na Universidade do Estado de Minas Gerais - UEMG. E-mail: mariaineslcr@hotmail.com ORCID: http://orcid.org/0000-0003-4900-5278

6.Enfermeira. Mestre em Enfermagem. Docente na Universidade do Estado de Minas Gerais- UEMG. E-mail: alinetsilva@yahoo.com.br ORCID: http://orcid.org/0000-0002-6364-8491

CONTATO: Gabriela da Cunha Januário | Rua Três de Maio, n 113 - Apto 204 - Centro | Passos | Minas Gerais | Brasil | Telefone: (37) 988534200 | E-mail: gabriela_cunha92@hotmail.com
\end{abstract}

COMO CITAR: Souza AC, Alves AC, Januário GC, Oliveira MEF, Ribeiro MILC, Silva AT. Percepções de um grupo de voluntários frente ao trabalho com pacientes oncológicos. R. Saúde Públ. Paraná. 2020 Jul;3(1):31-40. 
RESUMO Este estudo teve como objetivo analisar a percepção de um grupo de voluntários frente ao trabalho com pacientes oncológicos. Trata-se de uma pesquisa descritiva-exploratória, com abordagem qualitativa. A população do estudo foi constituída por voluntários do Expresso Alegria. Foram selecionados voluntários que vão frequentemente às visitas hospitalares, pelo menos uma vez ao mês e que não estejam em fase de treinamento. $A$ coleta de dados ocorreu após a aprovação do Comitê de Ética em Pesquisa, por meio de um questionário aberto. A investigação foi realizada em três etapas: reunião com o responsável do Expresso Alegria; abordagem individual para formalização do convite e coleta de dados. Foram entrevistados nove participantes. Para interpretação, os dados foram submetidos à análise de conteúdo de Bardin. A partir dos resultados foram elaboradas três categorias empíricas. O estudo apresentou a importância do trabalho voluntário no tratamento dos pacientes oncológicos, levando alegria, conforto e humanização ao ambiente.

PALAVRAS-CHAVE: Institutos de Câncer. Humanização da Assistência. Voluntários de Hospital.

\begin{abstract}
This study aimed to analyze the perception of a group of volunteers regarding the work with cancer patients. It is a descriptive-exploratory research, with a qualitative approach. The study population consisted of volunteers from Expresso Alegria. The volunteers selected were those who frequently go to hospital visits, at least once a month, and who are not undergoing training. Data collection took place after approval by the Research Ethics Committee, through an open questionnaire. The investigation was carried out in three stages: meeting with the head of Expresso Alegria; individual approach to formalize the invitation and collect data. Nine participants were interviewed. For interpretation, data were submitted to Bardin's content analysis. From the results, three empirical categories were elaborated. The study showed the importance of voluntary work in the treatment of cancer patients, as they bring joy, comfort and humanization to the environment.
\end{abstract}

KEYWORDS: Cancer Care Facilities. Humanization of Assistance. Hospital Volunteers.

\title{
INTRODUÇÃO
}

A

tualmente, as instituições hospitalares são compostas por profissionais de diversas áreas e especialidades, sendo ambientes de acolhimento e intervenção para pacientes que estão passando por um processo de adoecimento'. Para ampliar o acolhimento nestas instituições, foi criada a Política Nacional de Humanização (PNH), com o intuito de contagiar trabalhadores, gestores e usuários do Sistema Único de Saúde (SUS) com princípios e diretrizes da PNH; fortalecer iniciativas de humanização existentes e desenvolver tecnologias relacionais e de compartilhamento das práticas de gestão e de atenção².

Deste modo, visando o acolhimento e a minimização do sofrimento causado pela doença e dor dentro das instituições de saúde, surge o voluntariado, com o intuito de agregar um novo olhar diferenciado e 
contribuir na humanização, realizando atividades que proporcionam bem-estar aos pacientes e aos seus acompanhantes?

A Lei $n^{\circ}$ 9.608, de 19 de fevereiro de 1998, define o voluntariado como sendo uma atividade não remunerada prestada por alguém a uma entidade pública ou instituição sem fins lucrativos com objetivos cívicos, culturais, educacionais, científicos, recreativos ou de assistência, dissociada de qualquer obrigação de natureza trabalhista, previdenciária ou afim, regulamentada por um termo de adesão estabelecido entre a entidade e o prestador do serviço ${ }^{3}$.

Atividades voluntárias ocorrem com frequência em pessoas diagnosticadas com câncer, em vários estágios da doença, desde o diagnóstico, tratamento e momento posterior. Este tipo de atividade, de forma complementar, se mostra positiva e engrandecedora pois muitas vezes as pessoas que participam já vivenciaram situações análogas em sua família e, por isso, podem dar depoimentos e tornarem-se incentivadoras da busca por tratamento ${ }^{4-5}$.

Neste contexto, a realização de atividades lúdicas e a terapia do riso proporcionadas pelos voluntários denominados doutores-palhaços pode significar a forma mais eficaz para diminuir o estresse da internação, uma vez que os sentimentos como temores, frustração, ansiedade e raiva, oriundos, principalmente, da doença e da internação sejam expressos tornando o ambiente hospitalar menos traumatizante e mais alegre. auxiliando no processo de adaptação do paciente oncológico ao tratamento ${ }^{5-6}$.

A literatura aponta que os benefícios não são somente para os pacientes, mas também para os voluntários que se dedicam a ajudar, promovendo nestes sujeitos efeitos positivos de comportamentos prósociais?. Portanto este estudo teve como objetivo analisar a percepção do trabalho voluntário do Expresso Alegria na atenção aos pacientes em tratamento oncológico.

\section{MÉTODO}

Trata-se de um estudo descritivo-exploratório, com abordagem qualitativa, realizado no Expresso Alegria, onde acontecem os encontros e cursos para a formação de Doutor Palhaço, localizado em uma cidade do sul de Minas Gerais. O Expresso Alegria é uma organização sem fins lucrativos, que tem como principal objetivo promover brincadeiras, entretenimento e fazer com que as pessoas deixem de lado momentos de tristeza e passem a sorrir, mas naturalmente. A organização possui em média 40 participantes.

Os Doutores Palhaços são um grupo liderado por ex-profissionais de circo, teatro e atividades ligadas à cultura, que realizam visitas à Santa Casa de Misericórdia de Passos, Lar São Vicente de Paulo, escolas, igrejas, creches, casa de recuperação de dependentes químicos e instituições ou entidades.

Foram incluídos no estudo os voluntários ativos, que vão frequentemente às visitas hospitalares, pelo menos uma vez ao mês, e foram excluídos aqueles que estavam em formação e que não estavam capacitados para a abordagem hospitalar. Ao final, participaram desta pesquisa nove voluntários do Expresso Alegria, experientes na atuação com pacientes oncológicos.

Os dados foram coletados entre os meses de agosto e setembro de 2018 por meio de um questionário aberto elaborado pelo próprio pesquisador, e também por um formulário contendo variáveis sociodemográficas dos voluntários (sexo, faixa etária, estado civil, filhos, raça, religião, escolaridade, entre outras). A investigação foi realizada em três etapas. Inicialmente, foi marcada uma reunião com o responsável pelo Expresso Alegria 
para liberação da equipe na participação do estudo. Em seguida, foi feita abordagem individual dos voluntários para formalizar o convite, apresentar o objetivo do estudo e, na terceira etapa foi realizada a coleta de dados. 0 término da coleta ocorreu na medida em que as informações se tornaram reincidentes, não resultando em novos achados. A análise destes dados foi realizada em outubro de 2018.

Para interpretação, os dados foram submetidos à análise de conteúdo de Bardin, que é constituída por três etapas: pré-análise, exploração do material e o tratamento dos resultados, inferência e a interpretação. A análise de conteúdo é definida como "um conjunto de técnicas de análise das comunicações visando a obter, por procedimentos sistemáticos e objetivos de descrição do conteúdo das mensagens, indicadores (quantitativos ou não) que permitam a inferência de conhecimentos relativos às condições de produção/ recepção" 8 .

A pesquisa foi aprovada $\left(n^{\circ} 2.666 .280\right)$ pelo Comitê de Ética e Pesquisa (CEP) e seguiu os preceitos da Resolução $n^{\circ} 466 / 2012^{9}$, envolvendo estudos com seres humanos. Os participantes assinaram o Termo de Consentimento Livre e Esclarecido (TCLE) em duas vias, podendo retirar seu consenso a qualquer momento.

\section{RESULTADOS E DISCUSSÃO}

Participaram do estudo nove voluntários. Destes, 6 (67\%) eram homens, com uma faixa etária variando entre 29 e 56 anos, 6 (67\%) participantes eram casados e 7 (78\%) tinham filhos. Analisando a escolaridade. 8 (89\%) possuíam ensino médio completo. A maioria dos participantes possuía renda pessoal variando entre 01 a 03 salários mínimos.

Analisando o tempo de atividade no Expresso Alegria, 6 (67\%) dos participantes desenvolvem o trabalho voluntário há mais de 04 anos e possuem uma frequência mensal nas atividades; destes, 3 (33\%) possuem outro familiar atuante no Expresso Alegria, sendo pais, sobrinhas, filhos e esposas, 3 (33\%) relataram possuir outro trabalho voluntário sendo: mutirão de casa própria, arrecadação de cestas básicas (mantimentos) e agasalhos, e distribuição de brindes e brinquedos em datas comemorativas

A Tabela 1 apresenta as características sociodemográficas dos voluntários participantes do estudo.

A partir dos resultados das falas dos participantes, foram elaboradas três categorias empíricas, sendo elas: motivos para ser voluntário; atuação como voluntário: atividades desenvolvidas e compromisso da equipe; e, auxílio no processo saúde e doença dos pacientes oncológicos. 
Tabela 1 - Distribuição dos participantes do estudo segundo o perfil sociodemográfico, Passos - MG. 2018. (n=9)

\begin{tabular}{|c|c|c|c|}
\hline VARIÁVEIS DO ESTUDO & CARACTERIZAÇÃO & $\mathbf{N}$ & (\%) \\
\hline \multirow{2}{*}{ Sexo } & Feminino & 3 & $33 \%$ \\
\hline & Masculino & 6 & $67 \%$ \\
\hline \multirow{4}{*}{ Faixa etária } & $18-30$ anos & 2 & $22 \%$ \\
\hline & 31-40 anos & 4 & $45 \%$ \\
\hline & $41-50$ anos & 1 & $11 \%$ \\
\hline & $>50$ anos & 2 & $22 \%$ \\
\hline \multirow{2}{*}{ Estado civil } & Solteiro & 3 & $33 \%$ \\
\hline & Casado & 6 & $67 \%$ \\
\hline \multirow{3}{*}{ Filhos } & O filhos & 2 & $22 \%$ \\
\hline & 1 filho & 4 & $45 \%$ \\
\hline & 2 filhos & 3 & $33 \%$ \\
\hline \multirow{3}{*}{ Raça } & Branco & 5 & $56 \%$ \\
\hline & Negro & 1 & $11 \%$ \\
\hline & Pardo & 3 & $33 \%$ \\
\hline \multirow{3}{*}{ Religião } & Católico & 2 & $22 \%$ \\
\hline & Evangélico & 5 & $56 \%$ \\
\hline & Não respondeu & 2 & $22 \%$ \\
\hline \multirow{5}{*}{ Escolaridade } & & 1 & $11 \%$ \\
\hline & Ensino fundamental incompleto & & \\
\hline & Ensino médio completo Ensino & 4 & $45 \%$ \\
\hline & $\begin{array}{l}\text { superior incompleto } \\
\text { Ensino superior completo }\end{array}$ & 2 & $22 \%$ \\
\hline & & 2 & $22 \%$ \\
\hline \multirow{3}{*}{ Renda pessoal } & 1 a 3 salários mínimos & 5 & $56 \%$ \\
\hline & 4 a 6 salários mínimos & 2 & $22 \%$ \\
\hline & > 6 salários mínimos & 2 & $22 \%$ \\
\hline \multirow{3}{*}{$\begin{array}{l}\text { Tempo de atividade no } \\
\text { Expresso Alegria }\end{array}$} & 1 a 3 anos & 2 & $22 \%$ \\
\hline & 4 a 6 anos & 6 & $67 \%$ \\
\hline & $>7$ anos & 1 & $11 \%$ \\
\hline \multirow{4}{*}{ Frequência no voluntariado } & Diariamente & 1 & $11 \%$ \\
\hline & Semanalmente & 2 & $22 \%$ \\
\hline & Quinzenalmente & 2 & $22 \%$ \\
\hline & Mensalmente & 4 & $45 \%$ \\
\hline \multirow{2}{*}{$\begin{array}{l}\text { Outro familiar atuante no } \\
\text { Expresso Alegria }\end{array}$} & Sim & 3 & $33 \%$ \\
\hline & Não & 6 & $67 \%$ \\
\hline \multirow{2}{*}{ Outro trabalho voluntário } & Sim & 3 & $33 \%$ \\
\hline & Não & 6 & $67 \%$ \\
\hline
\end{tabular}

Fonte: elaborado pelos autores (2018) 


\section{MOTIVOS PARA SER VOLUNTÁRIO:}

A motivação se caracteriza como uma grande inspiração que proporciona inúmeras expectativas, propagada por um desejo individual que leva o ser humano a exercer determinada atividade, entre elas o voluntariado1. Segundo um pesquisador brasileiro ${ }^{10}$, os motivos que levam as pessoas a fazerem trabalhos voluntários têm sido alvo de interesse de vários estudiosos. Entender a motivação dos trabalhadores pode ser importante para compreender quem busca o trabalho voluntário, as suas expectativas e, neste sentido, contribuir para melhor gestão de pessoas e dos serviços. Percebemos isso diante do relato dos participantes:

"Há algum tempo procurava por um trabalho voluntário mais consistente e organizado. Conheci o Expresso Alegria e me apaixonei, mas não dava certo fazer o curso por motivos de trabalho/viagens". V1 "Meu pai teve câncer e veio a falecer e nesse período eu conheci o Expresso Alegria e quis fazer parte". V2

"O motivo que me levou a participar é a felicidade em conseguir levar um pouco de bem-estar pra quem está passando por momentos difíceis". v9

Segundo autor referenciado', doar-se é realizar algo por alguém sem receber remuneração, é um gesto que qualifica e transforma a quem recebe estes cuidados e a quem doa o cuidado. $\mathrm{O}$ significar de tudo isso é tornar o momento agradável e confortável para o paciente.

Em um estudo ${ }^{11}$ realizado com voluntários em um hospital oncológico na Índia, afirma-se que as motivações que estas pessoas encontram para realizar o voluntariado estão na necessidade de poderem ajudar a sociedade, atendendo aos que sofrem. Outros aspectos positivos apontados são o trabalho em equipe, a oportunidade de compartilhar experiências e oferecer conforto e alegria ao próximo.

\section{ATUAÇÃO COMO VOLUNTÁRIO: ATIVIDADES DESENVOLVIDAS E COMPROMISSO DA EQUIPE:}

Segundo pesquisadores ${ }^{12}$, entende-se como voluntário a pessoa que, motivada pelos valores sociais de participação e solidariedade, doa seu tempo, trabalho, talento, de maneira espontânea e sem remuneração, o que beneficia tanto o trabalho da equipe médica como o próprio paciente. Cada voluntário desenvolve a atividade proposta dentro da equipe em que está inserido, como apresenta V6:

"Fazemos visitas no hospital beira leito ao paciente, levando a ele a arte do Clown pois ele internado não tem condições de ir a um teatro, um circo e é isso que levamos a ele, não só ao paciente mas ao acompanhante, familiares e funcionários do hospital, tentando amenizar aquele ambiente pesado das rotinas hospitalares e espalhando o ar da graça que é o trabalho fora do hospital levando a arte do Clown". V6

Estudo realizado ${ }^{13}$ sugere que Clown ou palhaço são termos com o mesmo sentido, mas distintos entre si. Clown é um representante de uma genealogia da comicidade que se caracteriza por criticar a sociedade e seus costumes. Geralmente a arte Clown enxerga o mundo a partir de uma visão particular, na qual traduz tudo o que vê de forma diferenciada e estas atitudes, muitas vezes, provocam o riso. Seu universo situa-se bem próximo ao da criança, o que justifica sua presença no hospital. Ainda segundo o mesmo autor, esta arte 
oferece à criança a oportunidade de contato com suas fantasias, inspirando sua criatividade, vislumbrando sua alegria, auxiliando-o na doença. Nas falas dos participantes V1 e V6 verificamos a importância de utilizar coisas lúdicas para alegrar o ambiente.

"No meu tempo livre, geralmente aos sábados, domingos e feriados sempre estou à disposição. Já me programo durante a semana e muitas vezes levo a família (quando é fora do hospital). Sempre estou renovando o repertório, o figurino, e estudando sobre o palhaço de hospital que me ensina muito para outras atividades de voluntariado e cada vez mais me torna uma pessoa melhor". V1

"Minha forma é mostrar que do mesmo jeito que as cores alegram (porque você pode pegar algo cinzento, algo preto e branco e se você colocar alguma cor viva, ela alegra), eu tento através das cores colorir a vida das pessoas, e deixo bem claro, até tem um coraçãozinho que eu dou de EVA todo colorido, já escrevo nele, que o amor de Deus possa colorir sua vida e uso meu nome de Dra. Colorida, e sempre faço algo usando cor, que é falar de arco-íris que foi a aliança que Deus fez, que Deus não vai abandonar essa aliança que foi selada por ele. E dessa forma estar falando, que não existe dia cinzento para quem sonha colorido". V3

Pesquisa $^{14}$ realizada apresenta em seus achados a importância de trabalhar com recursos como desenhos, pinturas, música e brinquedos, a fim de minimizar a experiência do adoecimento e hospitalização dos pacientes e familiares. Os participantes também levantaram outras atividades realizadas com o Expresso Alegria.

"O Expresso também desenvolveu o projeto Bolívia, no qual uma vez por ano arrecadamos o máximo de coisas que conseguirmos e essas coisas são levadas para comunidades ribeirinhas carentes, nessa viagem vão médicos, dentistas, voluntários e outras pessoas dispostas a ajudar as comunidades". V7

“Como voluntária, além das visitas, muitas vezes fazemos doação de cestas básicas e produtos de higiene para famílias e escolas carentes. Na maioria dos lugares que vamos nossas atividades são dançar, contar histórias, fazer piadas ou até mesmo sentar para conversar". v9

Segundo autores ${ }^{15}$, o trabalho voluntário pode ser visto como uma reação da sociedade civil organizada às lacunas que o estado deixa de preencher nas políticas públicas de bem-estar e, também, uma forma de manifestação de interesses contrários à postura meramente mercadológica. Em um estudo realizado por pesquisador brasileiro ${ }^{16}$ sobre a ONG Amigos do Bem, identificou-se que o trabalho voluntário favorece o desenvolvimento social da população. Esta capacidade está relacionada à capacidade dos voluntários em captar recursos, melhorando a vida da população local. Muitos participantes relataram a importância em compartilhar o amor, carinho e cuidado com o outro, mas, devido aos compromissos pessoais, nem sempre é possivel comparecer às visitas

[...] temos que respeitar a disponibilidade das pessoas. O grupo é muito eclético, temos voluntários que realmente honram seus compromissos e alguns poucos que destoam, nada demais". V1 "Poucos deles são empenhados. Alguns dedicam mais, podia ser mais, se doar mais". V8

Embora a atividade voluntária ocorra normalmente, mesmo com a falta de alguns voluntários durante as visitas, os participantes V3 e V6 reforçam a falta de compromisso de alguns colegas em relação ao trabalho. 
"Infelizmente pelo o que eu vejo são poucos que têm compromisso, porque são muitos os que fazem o curso, mas nós podemos contar nos dedos de uma mão quais são os que realmente vão nas visitas [...] sempre falta voluntário, talvez até pela dificuldade psicológica em estar lá, porque não é fácil, não é todo mundo que dá conta". V3

"A atividade voluntária é muito difícil pois exige tempo. A maior dificuldade que vejo é a falta de tempo, acaba que não temos o compromisso que deveríamos ter". V6

O interesse de alguém em realizar determinada tarefa, a princípio, pode ser definido por um processo psicológico complexo, resultante de uma relação entre o indivíduo e o ambiente que o circunda, ou ainda, um conjunto de forças energéticas, que atuam sobre o indivíduo, influenciando seu comportamento e determinando a sua forma, direção, intensidade e duração ${ }^{17}$. Uma forma de "driblar" a falta de compromisso de alguns voluntários é fazer com que trabalhos sejam realizados em grupos e que sempre tenham um outro trabalhador de suporte. Para isso é importante que mais pessoas se envolvam no voluntariado ${ }^{18}$.

Demais pesquisas ${ }^{19}$ acrescentam outro ponto que pode intensificar o descompromisso de alguns voluntários, como as dificuldades e conflitos encontrados no ambiente hospitalar. Neste contexto, é importante que a gestão hospitalar realize treinamentos com os profissionais, focando na importância do voluntariado para a instituição e pacientes.

\section{AUXÍLIO NO PROCESSO SAÚDE E DOENÇA DOS PACIENTES ONCOLÓGICOS: ATIVIDADES DESENVOLVIDAS E COMPROMISSO DA EQUIPE:}

Sabe-se que o cuidado aos pacientes oncológicos inclui a promoção da confiança entre o profissional e o paciente e o incentivo à participação dos familiares na assistência. Os profissionais da equipe de enfermagem, muitas vezes com o intuito de atenderem a demanda e a necessidade fisiológica do paciente, visando a recuperação de sua saúde, dedicam pouca ou nenhuma parte do seu tempo às questões psicológicas e sociais da pessoa hospitalizada, e é neste contexto que o trabalho dos voluntários torna-se essencial para o paciente oncológico ${ }^{20-21}$.

"[...] não levamos apenas alegria e sim bem-estar porque algumas vezes o que os pacientes querem é só um abraço ou alguém que os escute. [...] a gente cria um laço de amizades com eles".V2

"[...] Na maioria dos casos sempre muito bem, todos se divertem com a visita dos doutores palhaços, mas algumas vezes alguns pacientes preferem não receber visitas, por cansaço, dor ou mesmo por não gostarem e é claro que compreendemos a vontade dos pacientes. Os funcionários são sempre muito respeitosos conosco e geralmente os visitantes e pacientes adoram as brincadeiras, alguns nem querem nos deixar ir embora depois e quando estão internados a mais tempo, aguardam ansiosos pela visita na próxima semana. Muitas vezes acabamos criando um pouco mais de afinidade com alguns pacientes por visitá-los mais vezes. Já comemoramos até mesmo aniversários dos pacientes no hospital."V9

"[...] é um momento de levar bem-estar pra eles, um momento de distração, tentar conseguir sorrisos em meio à dor, tanto para o paciente como para o acompanhante, porque às vezes o acompanhante está presente, mas não consegue ajudar a distrair, não consegue passar um incentivo, um apoio, e nós vamos e mostramos que naquele momento difíil é possivel passar um pouco de bem-estar pra eles". V3

"Eu sinto que eles gostam muito. Por exemplo: um paciente falou uma vez: 'quando vocês entram no hospital o ar do hospital muda, a gente se sente feliz'; Outro exemplo: 'Uma mãe colocou uma criança no meu colo e disse que eu curaria o filho dela' isso é muito gratificante". V8 
Outros estudos ${ }^{19}$ evidenciam que o voluntariado no meio hospitalar traz benefícios como: o conforto psicológico e físico ao paciente durante a internação; a melhoria da comunicação, do ambiente, do clima, dos relacionamentos e postura comportamental dos colaboradores da organização em que atua; e, a contribuição econômica tanto com doações financeiras, quanto com doações de seus serviços sem nenhum tipo de remuneração.

Pesquisas adicionais ${ }^{22.23}$ acrescentam que o voluntariado melhora a humanização do atendimento pois oferece conforto e alegria aos usuários, minimiza o estresse e proporciona algum alívio relativo à doença ou ao processo de internação, e que a humanização deve ser ponto-chave para promoção do bem-estar do paciente, garantindo que ele seja observado como um ser biopsicossocial e espiritual, devendo ser atendido de modo holístico e não apenas centrado na doença.

Neste sentido, é imprescindível que a população e os gestores busquem cada vez mais o voluntariado nas instituições de saúde, a fim de minimizar o sofrimento causado pelas enfermidades aos pacientes e familiares.

\section{CONSIDERAÇÕES FINAIS}

Neste estudo foi possivel identificar que os voluntários do Expresso Alegria se mostraram aptos às atividades realizadas, não apresentando sinais de insatisfação com o cotidiano do trabalho voluntário. Eles promovem o entretimento através de músicas, brincadeiras, teatros, estimulando o raciocínio e interagindo com pacientes oncológicos visando transformar a internação hospitalar oncológica em um momento mais alegre e agradável, contribuindo positivamente para o bem-estar do paciente e sua recuperação.

Foi possivel contemplar as percepções dos voluntários que apresentaram satisfação por poder participar deste trabalho e imenso sentimento de gratidão vivenciado através do reconhecimento de suas atividades pelos pacientes. Observamos também que a fundamental essência motivadora do trabalho não reside apenas na intenção de provocar o riso ou assegurar momentos de alegria, mas reside no bem-estar de todos.

O estudo teve como limitação a não adesão de todos os voluntários atuantes na Instituição. Espera-se que novas pesquisas possam ser elaboradas visando a humanização da assistência aos pacientes oncológicos.

\section{REFERÊNCIAS}

1. Diogo JS. A importância do voluntário na instituição hospitalar: uma transformação social que auxilia na qualidade de vida e bem-estar do paciente. Psicologia.pt [Internet]. 2016 [citado 2019 Dez 16]. Disponível em: http://www.psicologia.pt/artigos/textos/A1043.pdf

2. Brasil. Ministério da Saúde. Política Nacional de Humanização. 1. ed. [Internet]. Brasília, 2013. [citado 2019 Nov 15]. Disponível em: https:// bvsms.saude.gov.br/bvs/publicacoes/politica_nacional_humanizacao_pnh_folheto.pdf

3. Brasil. Lei n 9.608, de 18 de fevereiro de 1998. Dispõe sobre o serviço voluntário e dá outras providências. Brasília. 1998 [citado 2019 Nov 16]. Disponivel em: http://www.planalto.gov.br/ccivil_03/leis/19608.htm

4. Crippa A, Isidoro T, Feijó AGS. Voluntariado e saúde. Rev da AMRIGS. [Internet]. 2014 [citado 2020 Nov 27];58(3):247-251. Disponível em: https://pesquisa.bvsalud.org/portal/resource/pt/biblio-878205

5. Caires S. et al. Palhaços de hospital como estratégia de amenização da experiência de hospitalização infantil. Psico USF. 2014;19(3):377386.

6. Utsunomiya KF. et al. Mad Alegria: Palhaços de hospital. Rev Med, São Paulo. 2012;91(3):202-208. VOLUNTARIADO DAS NAÇÕES UNI- 
DAS. Relatório do Estado do Voluntariado no Mundo. 2011.

7. Miller JG, Kahle S.Hastings PD. Roots and benefits of costly giving: children who are more altruistic have greater autonomic flexibility and less family wealth. Psychol Sci [Internet]. 2015 [citado 2020 Mai 27]:26(7). Disponível em: https://journals.sagepub.com/doi/ abs/10.1177/0956797615578476

8. Bardin L. Análise de conteúdo. 6. ed. [Internet]. Lisboa, 2011. [citado 2019 Nov 22]. Disponível em: https://www.ets.ufpb.br/pdf/2013/2\%20 Metodos\%20quantitat\%20e\%20qualitat\%20\%20IFES/Livros\%20de\%20Metodologia/10\%20\%20Bardin,\%20Laurence\%20\%20 An\%C3\%A1lise\%20de\%20Conte\%C3\%BAdo.pdf

9. Brasil. Ministério da Saúde. Conselho Nacional de Saúde. Resolução n 466 de 12 de dezembro de 2012. Dispõe sobre os aspectos éticos e legais envolvendo pesquisa com seres humanos. [Internet]. Brasília: publicada no DOU n.12, quinta-feira, 13 de junho de 2013 - Seção I - p. 59. 2013. [citado 2019 Nov 3]. Disponível em: https://bvsms.saude.gov.br/bvs/saudelegis/cns/2013/res0466_12_12_2012.html

10. Assis KA. Doutores-palhaços: coping de trabalhadores voluntários em ambiente hospitalar. 42f. Monografia (Graduação em Psicologia) - Universidade Católica de Brasília. 2017.

11. Muckaden MA, Pandya SS. Motivation of volunteers to work in palliative care setting: A qualitative study. Indian J Palliat Care [Internet] 2016 [citado 2020 Jan 4];22(3). Disponivel em: http://www.jpalliativecare.com/article.asp?issn=0973-1075;year=2016; volume=22;issue=3;sp age=348;epage=353;aulast=Muckaden

12. Oliveira ER. Impacto do trabalho voluntário nos níveis de empatia, autoestima e bem-estar subjetivo. Dissertação (Programa de PósGraduação em Psicologia Social) - Universidade do Estado do Rio de Janeiro. 2018.

13. Santos EB. Nariz vermelho: fundamentos do Clown na ação da UT Riso. 86f. Monografia (Faculdade de Teatro) - Universidade Federal de Sergipe. 2013

14. Castro DP. Andrade CUB, Luiz E, Mendes M, Barbosa D, Santos LHG. Brincar como instrumento terapêutico. Pediatria [Internet]. 2010 [citado 2020 Jan 23];32(4). Disponivel em: file:///C:/Users/Usu\%C3\%A1rio/Downloads/BrincarparaestimularartigopublicadoFALTAIMPRIMIRECOLOCARNAPASTA.pdf

15. Paz MB, Costa TTP. Voluntariado: o amor não acaba para quem é do bem. Memória do projeto experimental (Faculdade de Comunicação) - Universidade de Brasília. 2018.

16. Moreno Junior OL. Gestão do voluntariado em organizações não governamentais: uma aplicação do método de estudo de caso na ONG Amigos do Bem. 189f. Dissertação (Mestrado em Administração) - FACCAMP. 2014.

17. Krieger S. Explorando as relações entre altruísmo, bem-estar subjetivo e esquemas iniciais desadaptativos. Dissertação (Programa de Pós Graduação em Psicologia Social) - Universidade do Estado do Rio de Janeiro, Rio de Janeiro. 2016.

18. Flores DO, Sousa EG. Governança Corporativa no Terceiro Setor - Um Estudo de Caso. Encontro de Gestão e Negócios (EGEN), 2016. Disponivel em: http://www.poncedaher.net.br/egen/sites/default/files/gestao-social-2016-OK-16- 31.pdf

19. Rodrigues KB, Meyer Júnior V. Cruz JAW. Trabalho voluntário e seu gerenciamento: desafios para um hospital comunitário. RAHIS. [Internet]. 2014 [citado 2020 Jan 20];11(4).Disponivel em: https://revistas.face.ufmg.br/index.php/rahis/article/view/2179

20. Jansen MF Santos RM, Favero L. Benefícios da utilização do brinquedo durante o cuidado de enfermagem prestado à criança hospitalizada. Rev Gaúcha Enferm. [Internet]. 2010 [citado 2019 Nov 20];31(2). Disponível em: https://www.scielo.br/scielo.php?script=sci_ arttext\&pid=S1983-14472010000200007\&lng=pt\&tlng=pt

21. Maia EBS, Ribeiro CA, Borba RIH. Understanding nurses awareness as to the use of therapeutic play in child care. Rev Esc Enferm. USP.[Internet]. 2011 [citado 2019 Nov 10];45(4).Disponínel em: https://www.scielo.br/scielo.php?script=sci_arttext\&pid=S0080-62342011000400007

22. Martins MCFN, Bersusa AAS, Siqueira SR. Humanização e voluntariado: estudo qualitativo em hospitais públicos. Rev Saúde Publ. [Internet]. 2010 [citado 2019 Nov 11];44. Disponivel em: https://www.scielo.br/scielo.php?pid=S0034-89102010000500020\&script=sci_ arttext\&tlng=en

23. Silva CPR, Conceição AP, Chagas APS. Clown - o palhaço como intervenção e humanização em saúde. J Health Biol Sci. [Internet]. 2017 [citado 2020 Mai 27];5(4). Disponivel em: file:///C:/Users/Usu\%C3\%A1rio/Documents/1181-5734-1-PB.pdf

CONFLITO DE INTERESSE

Este trabalho não possui conflito de interesses.

RECEBIDO: 28/01/2020

ACEITO: 09/06/2020 